\title{
The Research of Multimedia Data Transmission Basis on the Inventory and Queuing Theory
}

\author{
Shen Jia-jie \\ Computer center of School of Information Science \& Technology \\ East china normal university \\ Shanghai, china, 18817519704 \\ Shenjiajie888@yahoo.cn
}

\begin{abstract}
Aim to problem how the packet size effect to the multimedia data transmission and how to design the packet size to reach the best efficient of transmission. The research is mainly to use the inventory and queuing theory find the best way to design the send packet size. Though the model is established and calculated, The best value have been given. The experiment result also proof that exist best point of the packet size .
\end{abstract}

Keyword-Multimedia transmission, inventory theory, Queuing theory, network efficiency

\section{Introduction}

The research of the packet size have been done by vary researcher. The basis question of packet size is how to design the packet size to fit the different situation of the network. In the paper, It mainly talking about the multimedia data transmission under the multimedia transmission condition.

In the Optimization theory, There are two theory about the Optimize store and queue. That is inventory and queuing theory. There are a lot of research , Theorem and results above them. So, the question of packet size can be transform to the inventory and queue's problem. If we truly know about the situation of network. We can use the formula to calculate the optimistic packet size of the network.

This paper will organized as follow: In the first section will introduce some related work. In third section, The models and experiment Method will be presented. In forth section, The experiment result will be given.

\section{II. related work}

There are already exists dazzle of papers discuss the how to built a network whatever in the condition of wireless[1][2][3] and multimedia[4][5][6]. They use a lot of method to ensure the Qos of the network under different situation

However, the most researchers focus on the normal simulate. In the paper we show the different perform of network.[1][2][3],the packet size's research is also basis on it[4][5]. Then the inventory and queuing theory is research by vary research, so the inventory and queuing theory[5][6] is research in range of disaster recover to the computer budget allocation.

So, It's still can be use to simulate the packet size of network, so we should fully use of it.

\section{Models and Experiment Method}

Thought there are a lot of question about this big question and also exist a lots of tools and models to solve this question. The model uses inventory and queuing theory. to finish the job.

Assumption 1 : there are three kinds of nodes in the whole network:

1. It is node that only receive the multimedia data.

2. It is node that only send the multimedia data.

3. It is node that both send and received the data.

Assumption 2 : Between each of the sender and receiver, there is a queue warehouse. Queue warehouse will change with time in collections.

Assumption 3 : The Delay time of the warehouse is almost equal.

Assumption 4 : The network is good enough that almost all the package can reach the target node. In another words, almost no package lost.(Note: with the development of the network will be satisfied . )

So, the whole network will become the warehouse of nodes the or the queue of them. Between the sender and receiver, there is a queue warehouse between each sender and receiver .

Follow is the figure of the architecture of the model.

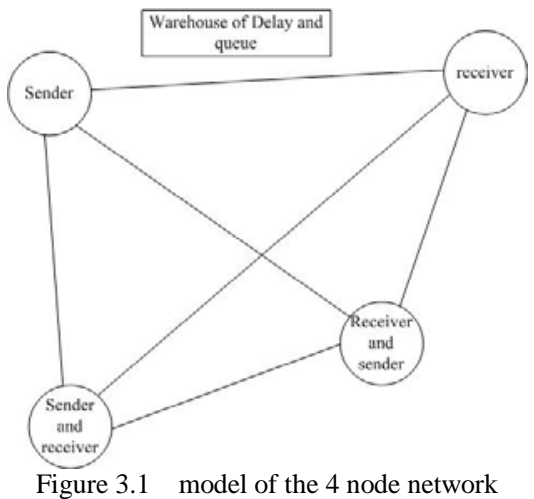

As the figure 3.1 ,Between two nodes there is a warehouse. Packet size is become the a quantity of one time provide, sender's send rate becomes the provide rate and receiver's nodes becomes the rate of consume. Also the delay time should be considered. 
Question to find the optimism Package size of network to make the network have most efficiency under no loss situation. So, the Constraint formula is:

$$
\left\{\begin{array}{c}
\text { min cost of network } \\
\text { obj not loss network } \\
\text { var package size }
\end{array}\right.
$$

Cost include two part:

1. Send a packet need some cost.

2. If the package in the network it will cost in the network and buffer.

So, the follow is how to decide the Optimistic the packet size in the send end basis on the inventory theory. the mainly difference between this model with others is that it is not focus on the particular route between the sender and receiver. The mainly advantage of it is to reduce the difficulty of the model.

Then, some definitions are given.

Definition 3.1: The whole network is a binary collections $(\mathrm{N}, \mathrm{W}), \mathrm{N}$ is the collection of senders or receivers' nodes, $\mathrm{W}$ is the collection of queuing warehouse between two nodes.

Definition 3.2: There are a queue warehouse between every two nodes and it have two queue can upload and download at the same time(but we only should consider one way).

Definition 3.3: The packet size is all possible packet size of send end. (marked as QS, QS $=(\forall x)(x \in S)$ (S is the all ))

Definition 3.4: Every warehouse have some delay between two end of queue warehouse and delay time between two end nodes is constant(as assumption 3) (marked as $T_{d}$ ).

Definition 3.5: Under the maximum transmission effect, The packet size is called as the best size of the warehouse.(marked as $Q_{0}, Q_{0} \in Q S$ )

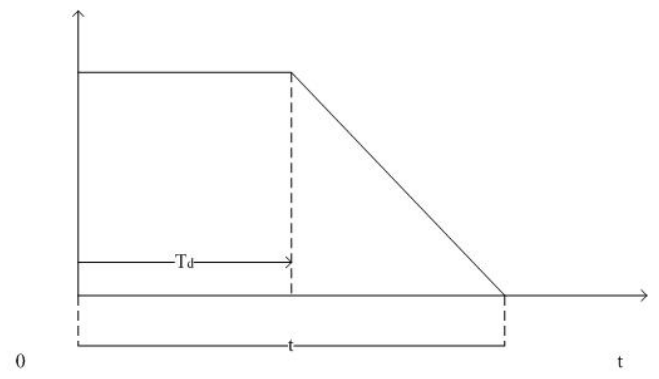

Figure 3.2 Illustration of $\mathrm{t}$ in Theorem 3.1

Theorem 3.1 : The min warehouse size is change with delay time, sender and receiver. And if the sender's capacity is $\infty$ and not allows interrupt of the video transmission, the min size of the packet is
$Q_{0}=R\left(t_{0}-T_{d}\right)=\sqrt{\frac{2 C_{3} R}{C_{1}}-T_{d}^{2} R^{2}}-R T_{d}\left(C_{3}\right.$ is the cost of the every time the connection created, $C_{1}$ is cost of the warehouse store.).

Proof :

Let us suppose the storing cost is $\mathrm{R}$, marked as $\mathrm{Q}, \mathrm{Q}=\mathrm{Rt}$, the created cost is $C_{3}$, So the total cost is $C_{3}$, the time averaged cost is $\frac{C_{3}}{t}$

The average store $\frac{1}{t} \int_{0}^{T} R t d T=\frac{1}{2} R T^{2}, \quad$ so cost of average store is $\frac{1}{2} C_{1} R T^{2}+C_{1} R F T T_{d}$.

The total cost of the transmission is $C(t)=C_{3}+\frac{1}{2} C_{1} R T^{2}+C_{1} R T_{d} T$

$\frac{C(t)}{t}=\frac{C_{3}}{t}+\frac{1}{2} C_{1} R\left(t-T_{d}\right)^{2} \frac{1}{t}+C_{1} R T_{d}\left(t-T_{d}\right) \frac{1}{t}$

Let: $\frac{d(C(t) / t)}{d t}=-\frac{C_{3}}{t^{2}}+\frac{1}{2} C_{1} R+\frac{C_{1} R T_{d}^{2}}{2 t^{2}}=0$

So: if let the cost $t_{0}=\sqrt{\frac{2 C_{3}}{C_{1} R}-T_{d}^{2}}$ (for3.1.1)

Use take for 3.1.1 to:

$Q_{0}=R\left(t_{0}-T_{d}\right)=\sqrt{\frac{2 C_{3} R}{C_{1}}-T_{d}^{2} R^{2}}-R T_{d}$

(for3.1.2).(Note: $T_{d}>=\sqrt{\frac{2 C_{3}}{C_{1} R}}$ is the monotonically discreasing function of $\mathrm{Q}$

Proof: $T_{d}=\sqrt{\frac{2 C_{3}}{C_{1} R}} \quad \mathrm{f}=\frac{1}{2} C_{1} R t$ is the increasing function it also can be proof in the $T_{d}<\sqrt{\frac{2 C_{3}}{C_{1} R}}$ )

Q

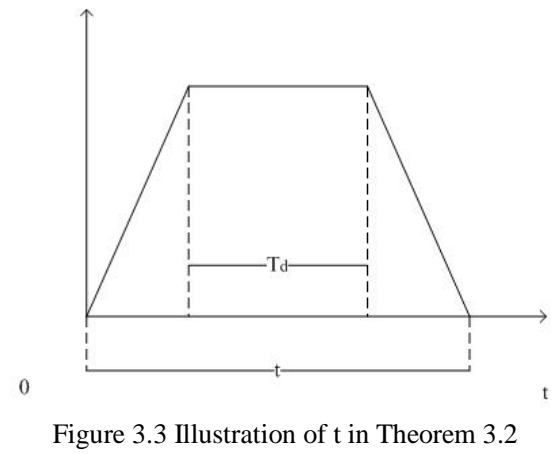

Theorem 3.2 : if the sender's capacity is $\mathrm{R}$ and not allows interrupt of the video transmission, the min size of the 
$Q=R\left(\frac{t_{1}-T_{d}}{2}\right)=\frac{1}{2} \sqrt{\frac{4 C_{3} R}{c_{1}}-T_{d}^{2} R^{2}}-\frac{1}{2} R T_{d}\left(C_{3}\right.$ is the cost of the every time the connection created, $C_{1}$ is cost of the warehouse store.)

Proof :

As the Theorem 1, Let us suppose the storing cost is R, marked as $\mathrm{Q}, \mathrm{Q}=\mathrm{Rt}$, the created cost is $C_{3}$, So the total cost is $C_{3}$, the time averaged cost is $\frac{C_{3}}{t}$

The storing cost is $C_{1} R T^{2}+C_{1} R T_{d} T$.

The total cost of the transmission is $C(t)=C_{3}+C_{1} R T^{2}+C_{1} R T_{d} T$

$C(t)=C_{3}+C_{1} R\left(\frac{t-T_{d}}{2}\right)^{2}+C_{1} R T_{d}\left(\frac{t-T_{d}}{2}\right)$

$\frac{c(t)}{t}=\frac{C_{3}}{t}+\frac{1}{t} C_{1} R\left(\frac{t-T_{d}}{2}\right)^{2}+\frac{1}{t} C_{1} R T_{d}\left(\frac{t-T_{d}}{2}\right)$

$\frac{d(c(t) / t)}{d t}=-\frac{c(t)}{t^{2}}+\frac{c_{1} R}{4}+\frac{c_{1} R T_{d}}{4 t^{2}}=0$

$t_{0}=\sqrt{\frac{4 c_{3}}{c_{1} R}-T_{d}^{2}}$ (for 3.2.1),

Take 4.1.1

to: $Q=R\left(\frac{t_{1}-T_{d}}{2}\right)=\frac{1}{2} \sqrt{\frac{4 C_{3} R}{C_{1}}-T_{d}^{2} R^{2}}-\frac{1}{2} R T_{d}$ (for3.2.2.)( Note: As Theorem 3.1, $T_{d}>\sqrt{\frac{4 C_{3}}{C_{1} R}}$ is the monotonically decreasing function of $\mathrm{Q})$.

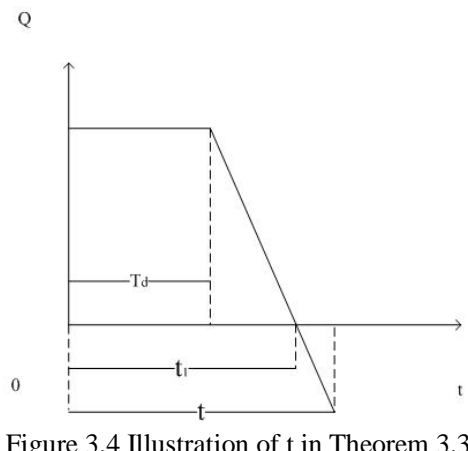

Theorem 3.3 : If the sender ' $\mathrm{s}$ capacity is $\mathrm{R}$ and not allows interrupt of video transmission, the min size of the packet is

$$
Q=R\left(t_{1}-T_{d}\right)=R\left(\sqrt{\frac{\left(2 C_{3}-C_{1} R T_{d}^{2}\right) C_{2} R}{C_{1}\left(C_{1} R+C_{2} R\right)}}-T_{d}\right) \text { Proof : }
$$

As the Theorem 1, Let us suppose the storing cost is $\mathrm{R}$, marked as $\mathrm{Q}, \mathrm{Q}=\mathrm{rt}$, created cost is $C_{3}$, So the total cost is $C_{3}$, the time averaged cost is $\frac{C_{3}}{t}$

The storing

cost is: $\frac{1}{2} C_{1} R T_{d}\left(t_{1}-T_{d}\right)+\frac{1}{2} C_{1} R\left(t_{1}-T_{d}\right)^{2}$

The penalty cost is $\frac{1}{2} C_{2} R\left(t-t_{1}\right)^{2}$

So,the total cost is that

$$
\begin{aligned}
& C(t)=C_{3}+\frac{1}{2} C_{1} R T_{d}\left(t_{1}-T_{d}\right) \\
& +\frac{1}{2} C_{1} R\left(t_{1}-T_{d}\right)^{2}+\frac{1}{2} C_{2} R\left(t-t_{1}\right)^{2} \\
& \frac{\partial)\left(c\left(t, t_{1}\right) / t\right)}{\partial t}=\frac{C_{1} R t_{1}}{t}-C_{2} R+\frac{C_{2} R t_{1}}{t}
\end{aligned}
$$

$t_{1}=\frac{C_{2} R t}{C_{1} R+C_{2} R}$

$$
\begin{aligned}
& \frac{\partial\left(c\left(t, t_{1}\right) / t\right)}{\partial t}=-\frac{C_{3}}{t^{2}} \\
& -\frac{\left(C_{1} R+C_{2} R\right) t_{1}^{2}-C_{1} R T_{d}^{2}}{2 t^{2}}+\frac{C_{2} R}{2}
\end{aligned}
$$

And let $t_{1}=\frac{C_{2} R t}{C_{1} R+C_{2} R}($ for 4.3 .1$)$

$\frac{\partial\left(c\left(t, t_{1}\right) / t\right)}{\partial t}=-\frac{C_{3}}{t^{2}}+\frac{\frac{C_{1} C_{2} R^{2} t^{2}}{C_{1} R+C_{2} R}+C_{1} R T_{d}^{2}}{2 t^{2}}=0$

$t=\sqrt{\frac{\left(2 C_{3}-C_{1} R T_{d}^{2}\right)\left(C_{1} R+C_{2} R\right)}{C_{1} C_{2} R^{2}}}$ (for4.3.2)

Take for 4.3.2

$t_{1}=t \frac{C_{2} R}{C_{1} R+C_{2} R}=\sqrt{\frac{\left(2 C_{3}-C_{1} R T_{d}^{2}\right) C_{2}}{C_{1}\left(C_{1} R+C_{2} R\right)}}$ (for4.3.3)

Finally, we will get

$Q=R\left(t_{1}-T_{d}\right)=R\left(\sqrt{\frac{\left(2 C_{3}-C_{1} R T_{d}^{2}\right) C_{2}}{C_{1}\left(C_{1} R+C_{2} R\right)}}-T_{d}\right)$

(for 4.3.3)

(Note: if $\quad C_{2} \rightarrow \infty \quad, \quad C_{1} R+C_{2} R \approx C_{2} R \quad$, $Q=R\left(t_{1}-T_{d}\right)=\sqrt{\frac{2 C_{3} R-C_{1} R^{2} T_{d}^{2}}{C_{1}}}-T_{d} R$ is the result of Theorem 3.1, As Theorem 3.1, $T_{d}>\sqrt{\frac{2 C_{3}}{C_{1} R}}$ is the monotonically decreasing function of Q).

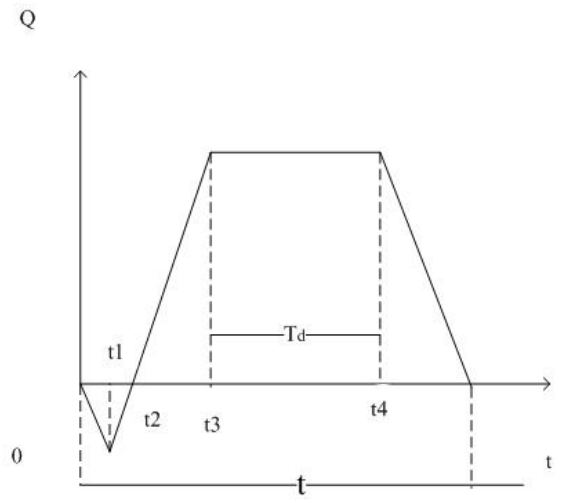

Figure 3.5 Illustration of $\mathrm{t}$ in Theorem 3.4 
Theorem 3.4: If the sender ' $\mathrm{s}$ capacity is $\mathrm{R}$ and not allows interrupt of video transmission, the min size of the packet is $Q=\frac{1}{2} R\left(\sqrt{\frac{\left(4 C_{3}-C_{1} R T_{d}^{2}\right) C_{2}}{C_{1}\left(C_{1} R+C_{2} R\right)}}-T_{d}\right)$

Proof:

Let $t_{23}+t_{34}+t_{4 e}=t_{1}$.

As the Theorem 3.3, Let us suppose the storing cost is R, marked as $\mathrm{Q}, \mathrm{Q}=\mathrm{rt}$, created cost is $C_{3}$, So the total cost is $C_{3}$, the time averaged cost is $\frac{C_{3}}{t}$

The storing cost is: $\frac{1}{2} C_{1} R T_{d}\left(t_{1}-T_{d}\right)+C_{1} R\left(\frac{t_{1}-T_{d}}{2}\right)^{2}$

The penalty cost is $C_{2} R\left(\frac{t-t_{1}}{2}\right)^{2}$

$$
\begin{aligned}
& C(t)=C_{3}+\left(C_{1} R\left(\frac{t_{1}-T_{d}}{2}\right)^{2}+C_{1} R \frac{T_{d}\left(t-T_{d}\right)}{2}\right) \\
& +C_{2} R\left(\frac{t-t_{1}}{2}\right)^{2} \\
& \frac{\partial\left(c\left(t, t_{1}\right) / t\right)}{\partial t_{1}}=C_{1} R \frac{t_{1}}{2 t}-\frac{1}{2} C_{2} R+C_{1} R \frac{t_{1}}{2 t} \\
& =>t_{1}=\frac{C_{2} R t}{C_{1} R+C_{2} R}(\text { for } 4.4 .1) \\
& \text { Set: } t_{1}=\frac{C_{2} R t}{C_{1} R+C_{2} R}, \text { we get }
\end{aligned}
$$

$$
\frac{\partial\left(c\left(t, t_{1}\right) / t\right)}{\partial t}=-\frac{C_{3}}{t^{2}}+\frac{\frac{C_{1} C_{2} R^{2} t^{2}}{C_{1} R+C_{2} R}+C_{1} R T_{d}^{2}}{4 t^{2}}=0
$$$$
t=\sqrt{\frac{\left(4 C_{3}-C_{1} R T_{d}^{2}\right)\left(C_{1} R+C_{2} R\right)}{C_{1} C_{2} R^{2}}}(\text { for 4.4.2) }
$$$$
t_{1}=t \frac{C_{2} R}{C_{1} R+C_{2} R}=\sqrt{\frac{\left(4 C_{3}-C_{1} R T_{d}^{2}\right) C_{2}}{C_{1}\left(C_{1} R+C_{2} R\right)}} \text { (for.4.4.3) }
$$$$
Q=R \frac{t_{1}-T_{d}}{2}=\frac{1}{2} R\left(\sqrt{\frac{\left(4 C_{3}-C_{1} R T_{d}^{2}\right) C_{2}}{C_{1}\left(C_{1} R+C_{2} R\right)}}-T_{d}\right)
$$

(Note: as Theorem 3.3 , if the

$$
C_{2} \rightarrow \infty, C_{1} R+C_{2} R \approx C_{2} R \text {, }
$$

$Q=R\left(\frac{t_{1}-T_{d}}{2}\right)=\frac{1}{2} \sqrt{\frac{4 C_{3} R}{c_{1}}-T_{d}^{2} R^{2}}-\frac{1}{2} R T_{d}$ Is result of

Theorem 3.2 and As Theorem 3.1, $T_{d}>\sqrt{\frac{4 C_{3}}{C_{1} R}}$ is the monotonically decreasing function of $\mathrm{Q}$ a).

IV. Experiment Result

The result of the Theorem 1 and Theorem 2 is as figure 4.1 and figure 4.2, you can see there are exist a point of $t$ exists the lowest cost of the network. 4.1:

The result of Theorem 3.1 and Theorem 3.2 as figure $1:$

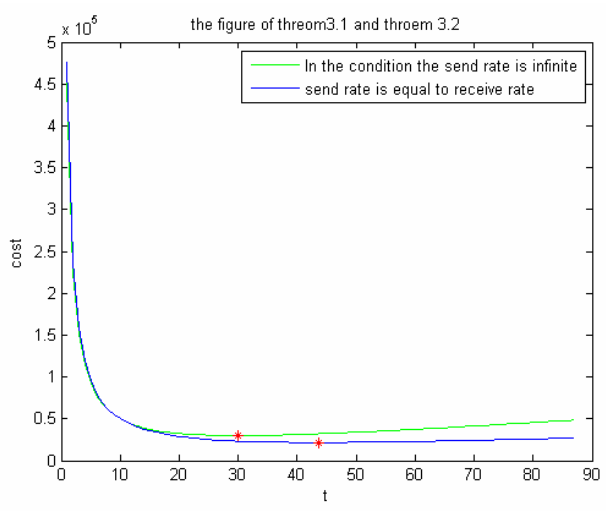

Figure 4.1 the result of theorem 3.13 .2 under $c 1=100$ and $c 3=500000$ delay=10 $\mathrm{R}=10$

As the figure 4.1, you can see that the Curve of the cost based on the transmission times. As the figure , If the $t$ is too small that transmission cost of the network will be very high because of there are only have a little of time of it to transmit data. If the time is too long the packet will have too long transmission time in the network ,so it will increase the cost.

Also the result of Theorem 3 and Theorem 4 as figure 4.2(a),(b):As figure 4.2(a),(b) show the relation of $t$ and $t_{1}$ with the cost. Like figure 4.1 , if $\mathrm{t}$ is too small will increase the cost of the transmission and it also exist a best point of the $\left(t, t_{1}\right)$ as the theorem 3.3 and theorem 3.4.( it is not obviously )

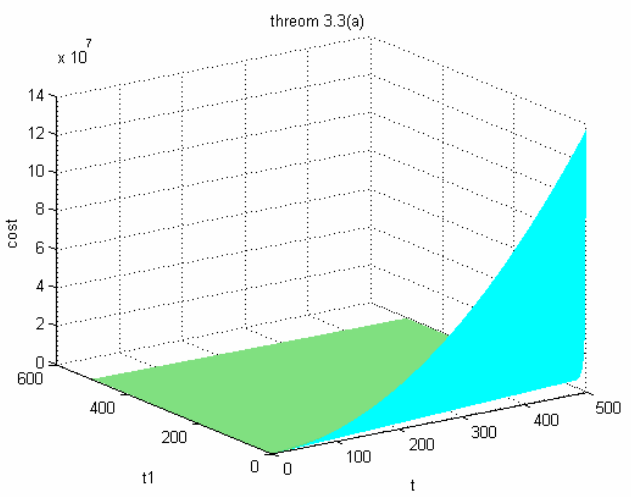

(a)theorem 3.3

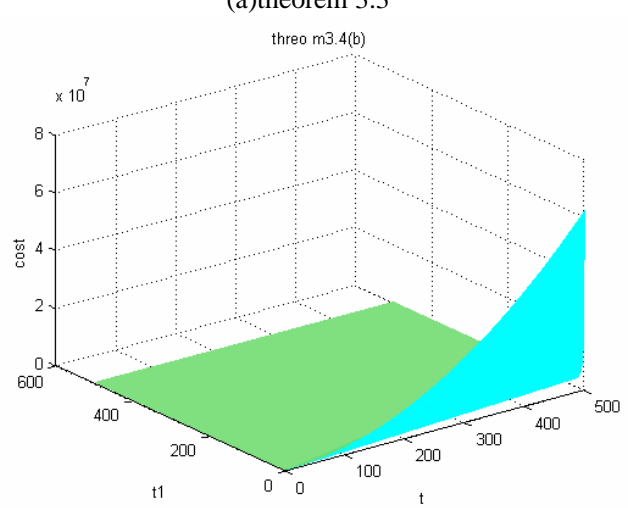

(b)theorem 3.4

Figure 4.2 the result of theorem 3.3 and 3.4 under $\quad$ c1=100 c2=1000 c3=100000 delay $=10 \mathrm{R}=1$

In order to have a more vividly view of Figure $4.2 \mathrm{n}$ if cost $>10000$ then let it equal to 10000 (as figure 4.2(a)(b)). 


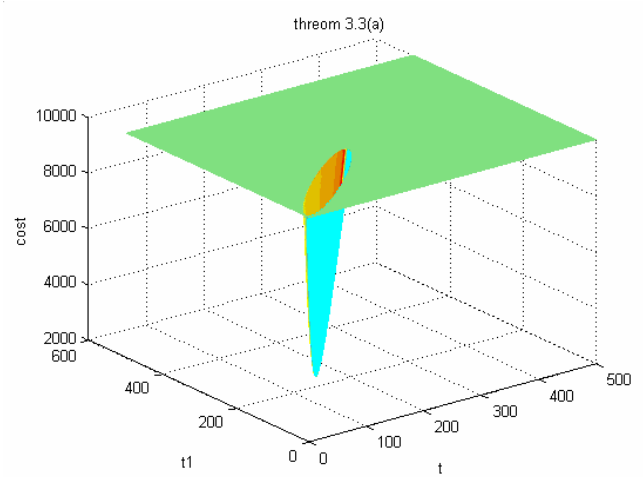

(a)theorem 3.3

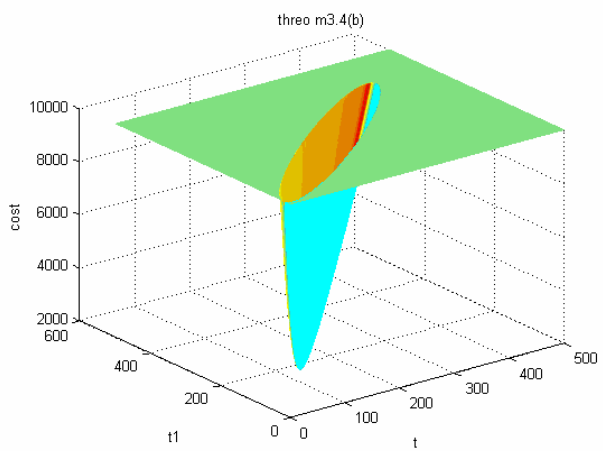

(b)theorem 3.4

Figure 4.3 the result after the procession cost $>10000$

The figure 4.3(a)(b) shows that the best value point of function which fits theorem 3.3 and 3.4.

\section{Conclusion}

In the paper, we calculate the best size of the packet basis on the inventory and queuing theory and result shows that it is simulate the different performs of the network when its packet size changes. It shows the different costs and perform of the network.and how to find the best point of the packet size.

It provide reference of host to change it packet size according to the protocol and environment of the network to reach the best efficient.

\section{Reference}

[1] H. Espeland1, C.l H. Lunde1, H. K. Stensland1, C. Griwodz, P. Halvorsen, Transparent Protocol Translation for Streaming,MM’07, September 23-28, 2007, Augsburg, Bavaria, Germany.

[2] G. Pan, H. Xue, Real Time Analysis of Current Transport Protocols in High Loss Networks, Beijing University of Posts and Telecommunications Beijing, China

[3] C. Bouras, G. Kioumourtzis, A. Gkamas, Simulcast Transmission for Video Applications : Performance Evaluation with an Integrated Simulation Environment, SPECTS 2009

[4] C. Dovroli, D. Moore, Packet-Dispersion Techniques and a Capacity-Estimation Methodology, TRANSACTIONS ON NETWORKING, VOL. 12, NO. 6, DECEMBER 2004

[5] J. Widmer, C. Boutremans, and J. L. Boudec, End-to-end Congestion Control for TCP-Friendly Flows with Variable Packet Size, ACM SIGCOMM Computer Communications Review, Volume 34, Number 2: April 2004

[6] A. D. Ridley, Michael C. Fu, FLUID APPROXIMATIONS FOR A PRIORITY CALL CENTER WITH TIME-VARYING ARRIVALS, Proceedings of the 2003 Winter Simulation Conference

[7] Y. M. Lee ,S. Ghosh ,Markus Ettl , SIMULATING DISTRIBUTION OF EMERGENCY RELIEF SUPPLIES FOR DISASTER RESPONSE ,Proceedings of the 2009 Winter Simulation Conference

[8] M. C. Fu, Chun-Hung Chen,SOME TOPICS FOR SIMULATION OPTIMIZATION, Proceedings of the 2008 Winter Simulation Conference 